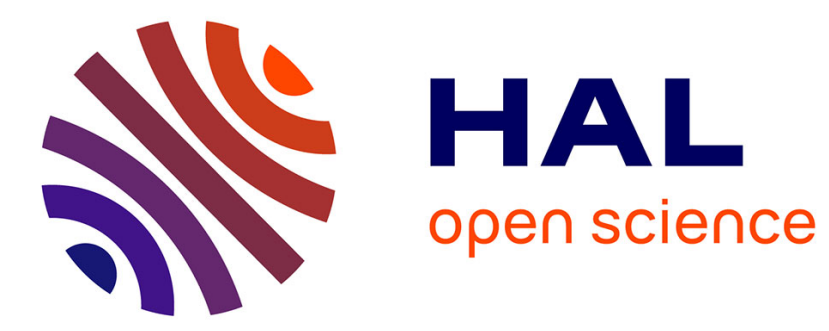

\title{
Optimal Sensor Placement in Road Transportation Networks using Virtual Variances
}

\author{
Enrico Lovisari, Carlos Canudas de Wit, Alain Kibangou
}

\section{To cite this version:}

Enrico Lovisari, Carlos Canudas de Wit, Alain Kibangou. Optimal Sensor Placement in Road Transportation Networks using Virtual Variances. CDC 2015 - 54th IEEE Conference on Decision and Control, Dec 2015, Osaka, Japan. hal-01185525

\section{HAL Id: hal-01185525 \\ https://hal.science/hal-01185525}

Submitted on 18 Sep 2015

HAL is a multi-disciplinary open access archive for the deposit and dissemination of scientific research documents, whether they are published or not. The documents may come from teaching and research institutions in France or abroad, or from public or private research centers.
L'archive ouverte pluridisciplinaire HAL, est destinée au dépôt et à la diffusion de documents scientifiques de niveau recherche, publiés ou non, émanant des établissements d'enseignement et de recherche français ou étrangers, des laboratoires publics ou privés. 


\title{
Optimal Sensor Placement in Road Transportation Networks using Virtual Variances
}

\author{
Enrico Lovisari, Carlos Canudas de Wit, and Alain Y. Kibangou
}

\begin{abstract}
This paper addresses the problem of Optimal Sensor Placement in Road Transportation Networks. The performance of the sensors is measured in terms of estimation error covariance of the Best Linear Unbiased Estimator of cumulative flows in the network over a long period. Sensors are to be placed in such a way that the sum of the error covariance and of a cost penalizing the number of sensors is minimized. The problem, inherently combinatorial, is relaxed using the concept of Virtual Variance. The resulting problem can be cast as a convex problem, whose computational load is much lower than the original combinatorial problem. Several variations are discussed, and the algorithm is applied to a regular grid network, for which an explicit comparison with the true optimum is offered, and, using data from the Grenoble Traffic Lab sensor network, to the real-world scenario of Rocade Sud in Grenoble, France.

Index Terms-Road Transportation systems; Flow reconstruction; Convex relaxation; Sensor placement.
\end{abstract}

\section{INTRODUCTION}

In the last decades the increase of the number of passenger and commercial vehicles has steered several crucial highways and arterial roads towards a state of near saturation, causing the emergence of periods of highly congested traffic on a daily basis [1]. Traffic congestion is responsible for the increase of travel times and for stop-and-go and other oscillatory phenomena, leading in turn to decreased safety, economical losses, and environmental and psychological hazards in terms of pollution and road rage [2]. Standard practice to solve congestion problem, by augmenting road capacity through extension or construction of highways and other arterial roads, is often infeasible due to physical constraints, roads passing through densely built up areas, and social opposition. Among alternative solutions, Intelligent Transportation Systems (ITSs) are expected to provide better and more robust techniques for real-time monitoring, prediction and actuation of traffic networks via exploitation of recent technological and theoretical advancements.

Of paramount importance is the ability to reconstruct the state of the network, as such an information that is used to forecast traffic evolution, to inform drivers in real-time through navigation systems, to provide statistical information to public authorities to detect in a timely fashion accidents and predict hazardous scenarios, and finally to compute controls and to actuate the network through traffic lights,

The authors are with Univ. Grenoble Alpes, Gipsa-Lab, with CNRS, Gipsa-Lab, and with Inria Grenoble Rhône-Alpes, F-38000 Grenoble, France enrico.lovisari, carlos.canudas-de-wit, alain.kibangoulgipsa-lab.fr. This work as been partially supported by the EU project SPEEDD FP7 n. 619435. ramp metering and speed limits, or, in the future, lane change and origin-destination suggestions [3], [4], [5], [6].

A primary source of information on the state of the network are fixed traffic detectors, namely, devices able to measure density, flow and average speed of vehicles crossing the section of the road where they are placed. Along with other sources of information, such as Floating Car Data, it can be used as input to observe the traffic state, such as the one designed in the companion paper [7].

Motivated by the importance of good quality monitoring, in this paper we address the Optimal Sensor Placement problem, namely, the problem of finding the best location where to physically place sensors. This is based on trading off between two contrasting objectives: the first, to maximize the performance of state reconstruction; the second, to minimize the total economic cost of the network.

The performance of the state reconstruction is usually related to the ability to properly estimate the density of vehicles in the roads. The latter is a time-varying quantity whose dynamics can be represented via partial differential equations models, such as the celebrated Lighthill-Whitham and Richards (LWR) model [8], or via their discretizations, as in the well known Cell Transmission Model [9]. Unfortunately, density reconstruction, and its relation with the network topology, is hindered by the complexity and nonlinearity of such macroscopic traffic models.

To simplify the setting, we consider the related problem of reconstruction in a static setting, by considering as performance metric the error covariance of an estimator of the cumulative flows in the network over a long period of time. Since the resulting trading off problem remains a combinatorial problem, we relax it using a method that we call Virtual Variance algorithm, based on the idea to associate to each sensor a virtual variance, which is large when the sensor is not needed for good reconstruction of the flow vector. We also discuss in detail two extensions of the proposed algorithm dealing with important scenarios, dealing with geographical constraints and budget limitations. The only input that the algorithm needs is an estimate of the matrix of splitting ratios and the nominal variance of each sensor. Since in real application a pre-existing sensor network is often unavailable, possible alternatives are field surveys with operators visually counting vehicles, as commonly done for calibration of traffic software, or temporary non-invasive equipment such as radar traffic detectors.

Optimal Sensor Placement is an ubiquitous problem that has received a high degree of attention in several communities due to its importance for network design. In Trans- 
portation Systems, it is of interest both in the dual-problem of best placement of hubs for cost-efficient transportation of goods and people [10] and Origin-Destination coverage [11], [12]. In these works, and differently from the present paper, the problem is cast as a mixed integer problem which corresponds to determine the minimal set of locations from which the flows on the whole network can be determined, and sensor measurements are assumed to be perfect.

The contributions of this paper are the following: 1) we formulate the Optimal Sensor Placement problem in terms of positions of sensors in a network of noisy sensors; 2) we propose the Virtual Variance algorithm as a relaxation of the (combinatorial) Optimal Sensor Placement problem; 3) we show the effectiveness of the devised technique on a regular grid, for which the true optimal placement is found by exhaustive search, and a real-world scenario consisting of the freeway "Rocade Sud" in Grenoble, France.

The rest of the paper is organized as follows: after presenting some notation, Section II formulates the Optimal Sensor Placement problem. Section III presents a solution based on the concept of Virtual Variance, while Section IV presents our two numerical experiments. Finally, Section V draws the conclusions and presents several future research directions.

\section{A. Notation}

The sets of real and positive real valued vectors of dimension $n$ are denoted $\mathbb{R}^{n}$ and $\mathbb{R}_{+}^{n}$, respectively. The set of real valued matrices of dimension $n \times m$ is $\mathbb{R}^{n \times m}$. $\mathbb{D}_{n} \subset \mathbb{R}^{n \times n}$ is the set of diagonal matrices of dimension $n \times n$. A positive definite (positive semidefinite, negative definite, negative semidefinite) matrix $A \in \mathbb{R}^{n \times n}$ is denoted $A>0(A \geq 0, A<0, A \leq 0)$, and for $A, B \in \mathbb{R}^{n \times n}$ $A>B$ means $A-B>0$ (and similarly for $\geq,<, \leq$ ). $A^{T}$ is the transpose of $A$. For a finite set $\mathcal{A}, \mathbb{R}^{\mathcal{A}}$ denotes the set of vectors indexed by elements of $\mathcal{A}$, and $|\mathcal{A}|$ its the cardinality. The identity matrix and the all-one vector (of suitable dimensions) are denoted $I$ and 1 , respectively.

A graph $\mathcal{G}$ is a pair $(\mathcal{V}, \mathcal{E}), \mathcal{V}$ being the set of nodes and $\mathcal{E}$ the set of edges. Edges are equipped with two functions $t: \mathcal{E} \rightarrow \mathcal{V}$ and $h: \mathcal{E} \rightarrow \mathcal{V}$, the head and tail functions, respectively, such that $e$ is though to the directed edge between $t(e)$ and $h(e)$. We allow for parallel edges, namely, edges having the same head and tail, but not for loops, namely, edges whose head and tail coincide. A path of length $n \geq 2$ is a sequence of edges $e_{1}, \ldots, e_{n}$ that are consecutive, namely, such that $\left.h\left(e_{i}\right)=t_{(} e_{i+1}\right)$ for $i=1, \ldots, n-1$. A path of length 1 is a path made of a single link.

\section{Problem Formulation}

We model a Transportation Network as a graph $\mathcal{G}=$ $(\mathcal{V}, \mathcal{E})$, in which junctions $v \in \mathcal{V}$ are interfaces between links, or cells, $e \in \mathcal{E}$. Origin cells, which carry vehicles in the network from the external world, and destination cells, which on the contrary let vehicles exit the network, will be referred to as onramps and offramps, respectively. We assume that for every cell there are at least one path from an origin to the cell, and a path from the cell to an offramp.
Let $\mathcal{O} \subseteq \mathcal{E}$ and $\mathcal{D} \subseteq \mathcal{E}$ denote the set of onramps and offramps, respectively. Vehicles flow through the cells of the network from their origin to their destination splitting at each junction according to deterministic splitting ratios. In particular, $R_{e j} \geq 0$ denotes the fraction of vehicles that exiting from cell $e$ want to enter into cell $j$. As such, $\sum_{j} R_{e j}=1$ if $e$ is not an offramp, and $\sum_{j} R_{e j}=0$ otherwise. We assume that the splitting ratios are fixed and perfectly known, and we gather them in a matrix $R=$ $\left[R_{e j}\right] \in \mathbb{R}^{n \times n}$ where $n=|\mathcal{E}|$.

The next sections are devoted to deriving some properties of the cumulative flows, to providing a simple linear model for the flows, and to formalizing the problem that we address.

\section{A. Flow linear constraints}

Let $f \in \mathbb{R}_{+}^{n}$ be the vector of cumulative flows, namely, the total flow, through the links of the network over a period of time $\left[t_{0}, t_{1}\right]$. Let $\rho_{e}(t)$ denote the density of vehicles in cell $e$ at time $t$, and $\ell_{e}$ the length of cell $e$. Then the definition of splitting ratios yields the relation $\ell_{e} \dot{\rho}_{e}(t)=$ $\sum_{j \in \mathcal{E}} R_{j e} \phi_{j}(t)-\phi_{e}(t)$ for each non onramp cell, where here $\phi_{e}(t)$ denotes the instantaneous flow through cell $e$. Integrating over $\left[t_{0}, t_{1}\right]$ yields $f_{e}=\int_{t_{0}}^{t_{1}} \phi_{e}(s) d s$ and

$$
\ell_{e}\left(\rho_{e}\left(t_{1}\right)-\rho_{e}\left(t_{0}\right)\right)=\sum_{j \in \mathcal{E}} R_{j e} f_{j}-f_{e}, \quad e \in \mathcal{E} \backslash \mathcal{O}
$$

Assume that $\left[t_{0}, t_{1}\right]$ is a long period of time and that at both times $t_{0}$ and $t_{1}$ the number of vehicles in the network is low, e.g., let $t_{0}$ and $t_{1}$ correspond to consecutive midnights. Then the magnitude of the elements of $\left\{\ell_{e}\left(\rho_{e}\left(t_{1}\right)-\rho_{e}\left(t_{0}\right)\right\}_{e \in \mathcal{E} \backslash \mathcal{O}}\right.$ is negligible compared with the cumulative flows in the network, and the following matrix relation holds approximately

$$
\bar{L} f \approx 0 \text {, }
$$

where $\bar{L} \in \mathbb{R}^{m \times n}, m=|\mathcal{E} \backslash \mathcal{O}|$, is obtained by removing from $L=R^{T}-I$ the rows corresponding to onramps.

As already mentioned in the Introduction, estimation of flows needs in principle to be coupled to a dynamical model for densities to realistically monitor a Transportation Network. Since it is however rather difficult to assess the performance of the observer of the density, in the present paper we simplify the problem by limiting our attention to the estimate of a vector of cumulative flow satisfying Eq. (1).

\section{B. Linear measurement model and the Optimal Sensor Placement problem}

We study in this section the performance of a linear estimator of the cumulative flows. Let $\mathcal{E}_{m} \subseteq \mathcal{E}$ be a generic set of cells in which sensors are placed, and consider the following simple linear measurement model

$$
y=H_{\mathcal{E}_{m}} f+\eta
$$

where

- $y_{s}$ is the measurement of the $s$-th sensor, namely $f_{e}+\eta_{s}$ if the $s$-th sensor is located on link $e$;

- $H_{\mathcal{E}_{m}} \in\{0,+1\}^{p \times n}, p$ being the number of sensors, with $\left[H_{\mathcal{E}_{m}}\right]_{s e}=1$ if the $s$-th sensor is located on link 
$e$, and $\left[H_{\mathcal{E}_{m}}\right]_{s e}=0$ otherwise, so that $H_{\mathcal{E}_{m}} \mathbf{1}=\mathbf{1}$ and $\mathbf{1}^{T} H_{\mathcal{E}_{m}} \mathbf{1}=p$;

- $\eta$ is a random noise vector with zero mean ${ }^{1}$ and covariance matrix $\Sigma_{\text {nom }}$. For sake of simplicity, we assume that noise components are independent with same variance $\sigma_{\text {nom }}^{2}$, so that $\Sigma_{\text {nom }}=\sigma_{\text {nom }}^{2} I$.

Let $V \in \mathbb{R}^{n \times r}, r=\operatorname{rank}\{\bar{L}\}$, be a matrix whose columns are an orthonormal basis of the right kernel of $\bar{L}^{T}$, i.e., $\bar{L}^{T} V=0$ and $V^{T} V=I$. Since $f$ belongs to the kernel of $\bar{L}$ by Eq. (1), there exists a vector $z \in \mathbb{R}^{r}$ such that $f=V z$. Therefore, we can write $y=H_{\mathcal{E}_{m}} V z+\eta$.

We consider a linear estimator $\hat{z}=K_{z} y+q_{z}, K_{z} \in \mathbb{R}^{r \times p}$ and $q_{z} \in \mathbb{R}^{r}$. The Best (minimum variance) Linear Unbiased Estimator of $z$ is then obtained solving

$$
\begin{array}{ll}
\min _{K_{z}, q_{z}} & \mathbb{E}\left[(z-\hat{z})(z-\hat{z})^{T}\right] \\
\text { s.t. } & \mathbb{E}[z-\hat{z}]=0 \\
& \hat{z}=K_{z} y+q_{z}
\end{array}
$$

Standard and straightforward computations show that (3) is equivalent to

$$
\begin{array}{ll}
\min _{K_{z}} & K_{z} \Sigma_{\text {nom }} K_{z}^{\prime} \\
\text { s.t. } & K_{z} H_{\mathcal{E}_{m}} V=I
\end{array}
$$

and $q_{z}=0$. The solution to the previous problem is

$$
K_{z}=\left(V^{T} H_{\mathcal{E}_{m}}^{T} \Sigma_{\text {nom }}^{-1} H_{\mathcal{E}_{m}} V\right)^{-1} V^{T} H_{\mathcal{E}_{m}}^{T} \Sigma_{\text {nom }}^{-1},
$$

with error covariance $\mathbb{E}\left[\left(\begin{array}{ll}z & -\hat{z}\end{array}\right)(z-\hat{z})^{T}\right]=$ $\left(V^{T} H_{\mathcal{E}_{m}}^{T} \Sigma_{\text {nom }}^{-1} H_{\mathcal{E}_{m}} V\right)^{-1}$. Consequently, the BLUE of $f$ is

$$
\hat{f}=K_{f} y=V\left(V^{T} H_{\mathcal{E}_{m}}^{T} \Sigma_{\text {nom }}^{-1} H_{\mathcal{E}_{m}} V\right)^{-1} V^{T} H_{\mathcal{E}_{m}}^{T} \Sigma_{\text {nom }}^{-1} y
$$

and its error covariance is

$$
\begin{aligned}
V_{p}\left(\mathcal{E}_{m}\right) & =\mathbb{E}\left[(f-\hat{f})(f-\hat{f})^{T}\right] \\
& =V\left(V^{T} H_{\mathcal{E}_{m}}^{T} \Sigma_{\text {nom }}^{-1} H_{\mathcal{E}_{m}} V\right)^{-1} V^{T} .
\end{aligned}
$$

The quantity $V_{p}\left(\mathcal{E}_{m}\right)$ depends on the matrix of splitting ratios and on the nominal variance of the sensors, two given quantities, and on the positions of the sensors, namely on $\mathcal{E}_{m}$. In the following, we use indeed $V_{p}\left(\mathcal{E}_{m}\right)$ as a metric to measure the performance of the placement $\mathcal{E}_{m}$. Clearly, with no additional constraint, the optimal placement is simply to equip every cell with a sensor. These devices, however, have a non-negligible purchase and maintenance cost, which has to be considered when designing a sensor network. In this paper, we make the simplifying assumption that the cost of a network over the lifetime of the network is proportional to the number of sensors via a coefficient $c>0$.

The problem that we want to address is the following Optimal Sensor Placement problem

$$
\min _{\mathcal{E}_{m}} \operatorname{trace}\left\{V_{p}\left(\mathcal{E}_{m}\right)\right\}+c\left|\mathcal{E}_{m}\right|
$$

The optimal solution of the previous problem trades off between network performance, measured as the trace of the

\footnotetext{
${ }^{1}$ The case of non-zero mean noise, for example due to more missed vehicles than overcounting, can be treated analogously. We consider the zero mean scenario for sake of simplicity.
}

estimator error covariance, and the total cost of the network: while the former tends to increase the number of deployed sensors, and also to position them properly, the latter aims to reduce it as much as possible.

The problem is inherently combinatorial, and the determining optimal positions of the sensors, namely, the optimal $\mathcal{E}_{m}$, becomes intractable even for very low network dimensions. In this paper we propose a different strategy, detailed in the next section, after a brief discussion on the minimum required number of sensors.

\section{On the minimum number of sensors}

Before proceeding, we quickly prove that the minimum number of sensors to estimate the vector of flows is equal to the number of onramps of the system. Assume by relabelling the cells that origin cells are the first $1, \ldots,|\mathcal{O}|$ cells. Then the matrix $L$ can be partitioned as

$$
L=\left[\begin{array}{cc}
-I & 0 \\
L_{o n} & L_{n n}
\end{array}\right]
$$

and notice that $\bar{L}=\left[\begin{array}{ll}L_{o n} & L_{n n}\end{array}\right]$. Consider the dual graph $\mathcal{G}^{d}=\left(\mathcal{V}^{d}, \mathcal{E}^{d}\right)$ in which $\mathcal{V}^{d}=\mathcal{E} \backslash \mathcal{O}$ and $(e, j) \in \mathcal{E}^{d}$ if $L_{e j} \neq 0$. Then it is easy to see that $L_{n n}^{T}$ is a sublaplacian of $\mathcal{G}^{d}$, namely, it is a Metzler matrix with $\sum_{j} L_{e j} \leq 0$. The following result is adapted from [13].

Lemma 1: Let $\mathcal{G}=(\mathcal{V}, \mathcal{E})$ be a graph and $J \in \mathbb{R}^{\mathcal{V} \times \mathcal{V}}$ be a weighted sublaplacian of $\mathcal{G}$. Then all the eigenvalues of $J$ have negative real part except possibly eigenvalues in 0 . Moreover, if $\mathcal{S}$ is the set of nodes $v$ for which $\sum_{u} J_{v u}<0$, then $J$ is Hurwitz if for every $u$ there exists a directed path in $\mathcal{G}$ from $u$ to a node $v \in \mathcal{S}$.

In our case, $J=L_{n n}^{T}$ and $\mathcal{S}$ is the set of cells directly following an origin cell. Since by assumption for every cell $e$ there exists an origin $j \in \mathcal{O}$ and a path from $j$ to $e$, then there must also exist a $k \in \mathcal{S}$ and a path from $k$ to $e$, so that the assumptions of Lemma 1 are thus satisfied. Therefore, $L_{n n}$ is Hurwitz and thus invertible, and in turn $\bar{L}$ is a full rank matrix, with rank $m=|\mathcal{E} \backslash \mathcal{O}|$, the number of non-onramp cells, and its kernel has rank $r=|\mathcal{O}|$, namely $\operatorname{rank}\{V\}=r=|\mathcal{O}|$.

\section{RELAXATION Via ViRTUAL VARIANCES}

It is easy to realize that links in which sensors are not present can be though of as links with sensors whose variance is infinite. Indeed, if by convention we consider $+\infty$ to be an admissible variance, the previous problem is equivalent to assigning a virtual variance $\sigma_{e}^{2}$ to each sensor, and decide for which it should be $\sigma_{e}^{2}=\sigma_{\text {nom }}^{2}$, and for which it should be $\sigma_{e}^{2}=+\infty$. With this interpretation, we can set $\mathcal{E}_{m}=\mathcal{E}$, so that $H_{\mathcal{E}_{m}}=I$.

Let $\Sigma$ be the (diagonal) matrix of virtual variances, and let the corresponding trace of error covariance be denoted, with an abuse of notation, $V_{p}(\Sigma)$. Our approach is based on the intuitive idea that by increasing the variance on the sensors that are not in the solution to Eq. (5) the quantity $V_{p}(\Sigma)$ does not increase much. 
More formally, we consider thus the following problem

$$
\begin{array}{ll}
\min _{\Sigma \in \mathbb{D}_{n}} & \operatorname{trace}\left\{V\left(V^{T} \Sigma^{-1} V\right)^{-1} V^{T}\right\}+f(\Sigma) \\
\text { s.t. } & \Sigma \geq \Sigma_{\text {nom }}
\end{array}
$$

where $f(\Sigma)$ is a decreasing function of the diagonal elements of $\Sigma$. Notice that $\Sigma$ is the optimization variable in (6): by reducing the virtual variances, namely, the diagonal elements of $\Sigma$, the trace of the error covariance decreases and the term $f(\Sigma)$ increases, and viceversa.

For sake of simplicity, let $f(\Sigma)=1^{T} \Sigma^{-1} \mathbf{1}$. With the change of variables $\Omega=\Sigma^{-1}, \Omega>0$ diagonal, and noticing that trace $\left\{V\left(V^{T} \Sigma^{-1} V\right)^{-1} V^{T}\right\}=\operatorname{trace}\left\{\left(V^{T} \Sigma^{-1} V\right)^{-1}\right\}$ by known properties of the trace and because $V^{T} V=I$, we obtain the following problem

$$
\begin{array}{ll}
\min _{\Omega \in \mathbb{B}_{n}} & \operatorname{trace}\left\{\left(V^{T} \Omega V\right)^{-1}\right\}+\gamma \mathbf{1}^{T} \Omega \mathbf{1} \\
\text { s.t. } & 0 \leq \Omega \leq \Sigma_{\text {nom }}^{-1}
\end{array}
$$

Once a solution $\Omega$ is found, we obtain the solution of (6) by $\Sigma=\Omega^{-1}$; then, we discard all links whose virtual variance is above a certain threshold. We refer to this procedure as the Virtual Variance algorithm. Notice that if the solution provides high virtual variances at locations where sensors are redundant then this is effectively a way to select the most important cells where to place sensors.

This is however not enough to solve the problem. As numerical simulations show, it is often the case that (7) is solved by assigning low virtual variances to all sensors of the network, rather than keeping it low in some of them and high in others. In order to enhance diversity between sensors, we enrich the cost with a further term that penalizes homogeneity and is reminiscent of works on dissensus (as opposed to consensus) in multi agent networks. Our choice in this paper is the following: let $W \in \mathbb{R}^{n \times n-1}$ be an orthonormal base of the subspace orthogonal to 1 . The additional considered term is then proportional to $e^{-1^{T} W^{*} \Omega 1}$, which is, as required, a function that decreases as the diagonal elements of $\Omega$ become more and more different. Following this idea, we propose the following optimization problem to solve the original combinatorial problem

$$
\begin{array}{ll}
\min _{\Omega \in \mathbb{D}_{n}} & \operatorname{trace}\left\{\left(V^{T} \Omega V\right)^{-1}\right\}+ \\
\text { s.t. } & 0 \leq \Omega \leq \sigma_{\text {nom }}^{-2} I
\end{array}
$$

which, notice, is convex in the diagonal entries of $\Omega$. Here $\gamma$, the total variance weight, and $\kappa$, the discrepancy weight, are tunable parameters. In particular, notice that high $\gamma$ indirectly penalizes the number of sensors, thus yielding to solutions with higher virtual variances at the expense of poor performance. As already mentioned, the cells over which a sensors is to be placed are those whose virtual variance, computed via $\Sigma=\Omega^{-1}$, is below a certain threshold. Numerical simulations have shown that the resulting virtual variances are distributed in a highly bimodal way, the low and the large ones being different by several orders of magnitude, thus making easy to distinguish among the two groups.

\section{A. Optimal Sensor Placement with Location constraints}

It can be the case, due for example to physical constraints, that some cells cannot be equipped with sensors. This section shows how to adapt the proposed approach to this case.

In particular, let $\mathcal{E}_{a m} \subseteq \mathcal{E},\left|\mathcal{E}_{a m}\right|=k$, be the subset of available cells, and let $H_{\mathcal{E}_{a m}} \in\{0,+1\}^{k \times n}$ be built as in Section II-B. Then the following problem

$$
\begin{array}{ll}
\min _{\Omega \in \mathbb{D}_{k}} & \operatorname{trace}\left\{\left(V^{T} H_{\mathcal{E}_{a m}}^{T} \Omega H_{\mathcal{E}_{a m}} V\right)^{-1}\right\}+ \\
\text { s.t. } & 0 \leq \Omega \leq \sigma_{\text {nom }}^{-2} I
\end{array}
$$

is a restriction of (8) once we constrain sensors to be placed on cells in $\mathcal{E}_{a m}$ only, and the diagonal entries of $\Omega$ are the inverse of the virtual variances on the cells in $\mathcal{E}_{a m}$. The matrix $W$ is defined as in the previous section, but with suitable dimension $(k \times k-1)$. As in the general problem, cells are chosen only if the corresponding virtual variance is below a certain threshold.

Remark 1: By the discussion in Section II-C, the minimum number of sensors is $r=|\mathcal{O}|$. As such, if $\left|\mathcal{E}_{e m}\right|<|\mathcal{O}|$ the problem (9) is not well posed and the solution will only have very high virtual variances. Clearly, such a solution is not acceptable and should be discarded.

\section{B. Optimal Sensor Placement with Number of Sensors con- straints}

The second scenario that we discuss consists in imposing a constraint on the maximum number of chosen sensor, which might be due to hard budget constraints.

Our solution is based on the following iterative approach:

- Initialization: set $\gamma(0)$ and $\kappa$ to chosen nonnegative values; $t_{\max }$ to the maximum number of iterations; $n_{\max }$ to the maximum number of sensors;

- At the $t$-th step

- Solve (8) with $\gamma(t)$;

- If the Virtual Variance algorithm yields a solution with number of sensors less than $n_{\max }$, or if $t \geq$ $t_{\max }$, stop;

- Otherwise, set $\gamma(t+1)=g(\gamma(t))$, where $g$ is an increasing function of its argument, and iterate.

Since, as discussed in the previous sections, $\gamma$ weights the penalty to low variances, by iteratively increasing it the solution to (8) tends to exhibit more and more high virtual variances, thus reducing the selected number of sensors.

Remark 2: Again by the discussion in Section II-C, the specified maximum number of sensors cannot be be less than $r=|\mathcal{O}|$. If this is not the case the algorithm either does not find a solution and simply cycles until the last iteration step $t_{\text {max }}$, or numerically finds a solution with an extremely high trace $\left\{\left(V^{T} H_{\mathcal{E}_{a m}}^{T} \Omega H_{\mathcal{E}_{a m}} V\right)^{-1}\right\}$.

\section{NUMERICAL EXPERIMENTS}

In this section we present the results of two numerical experiments. In the first, we solve the problem of Optimal Sensor Placement in a small (but not trivial) regular grid over which we run an exhaustive search, thus allowing 


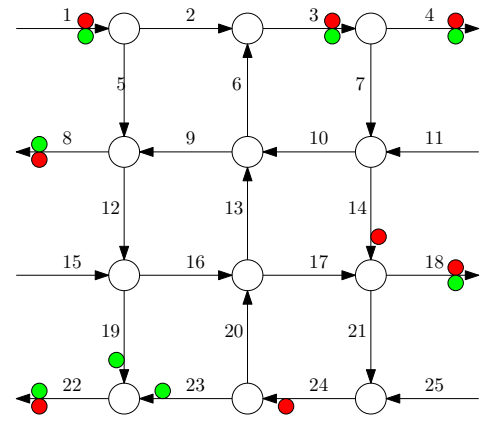

Fig. 1. The regular grid network used in the numerical experiment. The green dots correspond to the 8 cells selected by the Virtual Variance algorithm with $\gamma=2$ and $\kappa=20$. The red dots correspond to cells selected via exhaustive search when the number of possible sensors is 8 .

us to explicitly compare the result of the Virtual Variance algorithm with the optimal solution; in the second, we apply the procedure to the real-world case of the peri-urban freeway "Rocade Sud" in Grenoble, France.

\section{A. Regular grid}

We consider the 25 cells regular grid illustrated in Figure 1 . We assume that all sensors have a nominal variance of $\sigma_{\text {nom }}^{2}=1$ and that the cost of each sensor is $c=1$. First of all, we solve by exhaustive search the problem (5) for $\left|\mathcal{E}_{m}\right|=4,5,6, \ldots, 21$, considering for each possible number of sensors all possible combinations of cells and finding that which minimizes $V_{p}\left(\mathcal{E}_{m}\right)$. Then, we run the proposed Virtual Variance algorithm with total variance weight $\gamma=2$ and discrepancy weight $\kappa=20$, setting the lower threshold on the virtual variance for discarding a sensor to $T_{d}=100$.

The results are shown in Figures 1 and 2. In the former, we mark the 8 cells chosen by the Virtual Variance algorithm with a green dot, and the optimal cells when the number of possible sensors is 8 with a red dot. As it can be seen, both procedures place the majority of the sensors at the boundary of the network. The cost between the two solutions is not very different, as it can be observed in Figure 2. Here, we plot with crosses the cost $V(h)=$ trace $\left\{V_{p}(h)\right\}+$ ch for $h=4,5,6, \ldots, 21$, where $V_{p}(h)$ is $V_{p}\left(\mathcal{E}_{m}\right)$ with $\left|\mathcal{E}_{m}\right|=$ $h$ and $\mathcal{E}_{m}$ is the optimal placement. The optimal trade off between cost and performance is in this case 6 , as further increasing the number of sensors is not beneficial. The figure also shows that the solution of the Virtual Variance algorithm has 8 sensors, and plots as a circle the corresponding cost trace $\left\{V_{p}\right\}+8 c$. As it can be seen, not only the Virtual Variance algorithm finds a solution with a number of sensors that is close to the true optimum, but also, for that number of sensors, is able to place them almost in the optimal way.

\section{B. Rocade Sud}

Our second experimental setting is the Grenoble Traffic Lab (GTL) [14], a network of sensors deployed for monitoring and research purposes along the "Rocade Sud", a 12 $\mathrm{km}$ long freeway surrounding the town of Grenoble, France,

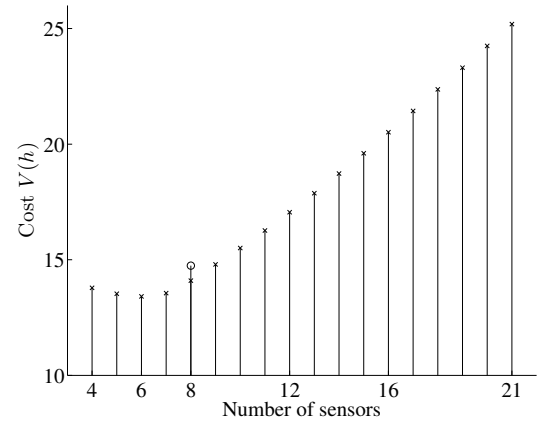

Fig. 2. Results of the exhaustive search and of the virtual variance algorithm. Crosses represent the cost of the true optimal placement of $4, \ldots, 21$ sensors. The Virtual Variance algorithm provides a solution with 8 sensors, with cost plotted as a circle.

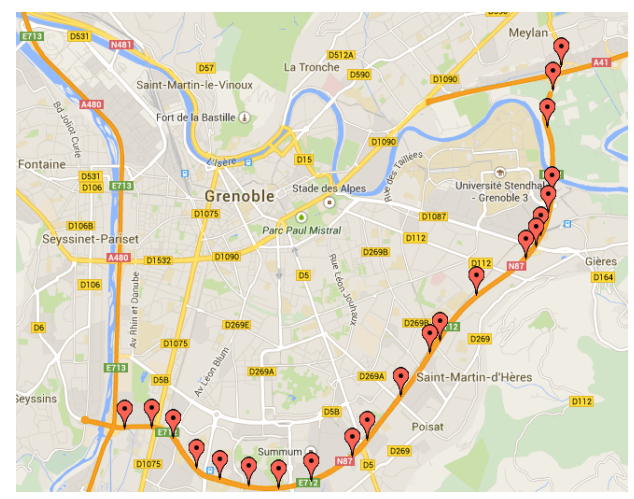

Fig. 3. The Grenoble Traffic Lab sensor network in Grenoble.

see Figure 3. The network consists in 135 magnetometers buried in the asphalt on both lanes of the main line and on each onramp and offramp, totalling 68 sensing locations. For our purposes, each sensing location will correspond to one sensor. For a detailed report on the GTL, we refer to [14].

The Rocade is partitioned in such a way that each cell includes one sensor; furthermore, for sake of simplicity, we do not consider onramps and offramp, limiting our attention to the main line. The corresponding network consists of 46 cells, and a stylized version of it is shown in Figure 4.

To estimate the matrix of splitting ratios $R$, we considered a period of data from the ten days 1st - 10th of April, 2014 (excluding the 5th and the 6th, weekend days), all typical working days, during the six hours time period 6:00 - 12:00. We gathered the cumulative measured flows in all 68 cells and we used them to estimate the matrix $R$ as follows: for each junction $v$, let $\mathcal{E}_{v}^{-}$and $\mathcal{E}_{v}^{+}$be its incoming and outgoing cells, respectively. Also, let $f_{v}^{-}(d)$ and $f_{v}^{+}(d)$ be the vectors of cumulative flows on the incoming and outgoing flows at junction $v$ for day $d$, namely, $f_{v}^{-}(d)=\left[f_{e}(d)\right]_{e \in \mathcal{E}_{v}^{-}}$, and analogously for $f_{v}^{+}(d)$. Let $F_{v}^{-}=\left[f_{v}^{-}(1), \ldots, f_{v}^{-}(8)\right]$ and $F_{v}^{+}=\left[f_{v}^{+}(1), \ldots, f_{v}^{+}(8)\right]$ be the collection in matrices of such flows. Also let $R_{v} \in \mathbb{R}^{\mathcal{E}_{v}^{-} \times \mathcal{E}_{v}^{+}}$be the local matrix of splitting rations, namely, let $\left[R_{v}\right]_{e j}$ be the splitting ratio of cell $e \in \mathcal{E}_{v}^{-}$towards cell $j \in \mathcal{E}_{v}^{+}$.

Then using Eq. (1) we have $R_{v}^{T} F_{v}^{-} \approx F_{v}^{+}$, so we can cast, for each $v \in \mathcal{V}$, the problem of estimation of the matrix of 


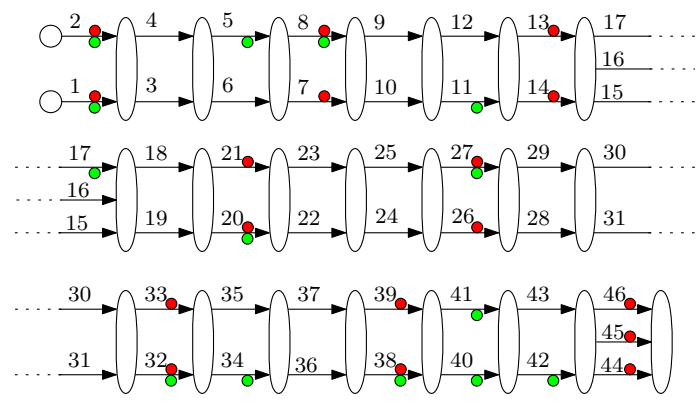

Fig. 4. Stylized representation of the main line part of the Rocade Sud. White ovals represents junctions of the graph. The selected positions of the 17 fixed sensors by CNIR are marked by red dots, those of the 14 sensors by the virtual variance algorithm by green dots.

splitting ratios as the optimization problem

$$
\begin{array}{ll}
\min _{R_{v}} & \left\|R_{v}^{T} F_{v}^{-}-F_{v}^{+}\right\|^{2} \\
\mathrm{s.t.} & R_{v} \mathbf{1}=\mathbf{1} \\
& {\left[R_{v}\right]_{e j} \geq 0, \forall e \in \mathcal{E}_{v}^{-}, j \in \mathcal{E}_{v}^{+}}
\end{array}
$$

The matrix of splitting ratios is then projected over a matrix consistent with the graph made of the cells on the main line only, simply deleting rows and columns corresponding to non-main line cells, and renormalizing the rows of the resulting matrix in such a way that the row sum is 1 .

We did not run an exhaustive search due to the relatively high dimension of the network. Instead, we compare the results with the locations of fixed loops installed for monitoring purposes by the Government Agency Centre national d'information routiére (CNIR) [15], which are placed on the cells marked with a red dot in Figure 4.

We run the Virtual Variance algorithm in three scenarios: 1) unconstrained scenario with total variance weight $\gamma=0.2$ and discrepancy weight $\kappa=20 ; 2$ ) unconstrained scenario with $\gamma=1$ and $\kappa=20 ; 3$ ) constrained scenario with number of sensors at most 10 , initial $\gamma=0.2$, and $\kappa=20$. We assume that $\sigma_{\text {nom }}^{2}=1$ and that the cost per sensor is $c=1$.

The results are summarized in Figure 4 and Table I. In the table we provide the optimal number of sensors computed via the Virtual Variance algorithm, as well as the corresponding estimator error covariance $V_{p}\left(\mathcal{E}_{m}\right)$ and the total cost $V\left(\mathcal{E}_{m}\right)=V_{p}\left(\mathcal{E}_{m}\right)+c\left|\mathcal{E}_{m}\right|$. In Figure 4, cells found in the unconstrained scenario with $\gamma=0.2$ are denoted using a green dot. As can be seen in Table I, our algorithm requires 3 sensors less than the network deployed by CNIR, while the error covariance increases only very slightly. In the constrained scenario and in the unconstrained scenario with high $\gamma$ (which, as explained above, indirectly penalizes the number of sensors), the error covariance $V_{p}\left(\mathcal{E}_{m}\right)$ increases, as expected. Interestingly, the chosen cells in the latter two cases are subsets of the cells chosen in the unconstrained case: in particular, in the constrained scenario all cells are kept except 8, 11, 20 and 41, and in the unconstrained scenario with high $\gamma$ the algorithm further discards cells 17 and 20. Whether this is a feature of the present case study or a more general property will be matter for future research.

\begin{tabular}{l|cccc} 
Scenario & $\gamma$ & $\begin{array}{c}\text { optimal } \\
\text { \# sensors }\end{array}$ & $V_{p}\left(\mathcal{E}_{m}\right)$ & $V\left(\mathcal{E}_{m}\right)$ \\
\hline Fix & & 17 & 3.8072 & 20.8072 \\
Unconstrained & 0.2 & 14 & 3.6867 & 17.6867 \\
Unconstrained & 1 & 8 & 5.6822 & 13.6822 \\
Constrained, \# $\leq 10$ & 0.43 & 10 & 4.6703 & 14.6703
\end{tabular}

TABLE I

RESULTS OF THE FOUR CONSIDERED SCENARIOS.

\section{CONCLUSIONS}

This paper addresses the problem of optimal placement of sensors in transportation networks. The problem, combinatorial by nature, is relaxed into a convex problem using the concept of Virtual Variance. Future research includes 1) addressing the case of uncertain matrix of splitting ratios, 2) providing theoretical guarantees on the robustness of the proposed approach, such as under sensor failure, and 3) investigating the scaling properties of the proposed strategy.

\section{REFERENCES}

[1] M. Papageorgiou, M. Ben-Akiva, J. Bottom, P.H.L. Bovy, S. P. Hoogendoorn, N. B. Hounsell, A. Kotsialos, and M. McDonald. ITS and traffic management. In C. Barnhart and G. Laporte, editors, Handbooks in Op. Res. and Manag. Sc.: Transportation, chapter 11, pages 715-774. Elsevier, 2007.

[2] J. Bilbao-Ubillos. The costs of urban congestion: estimation of welfare losses arising from congestion on cross-town link roads. Transport. Res. A-Pol., 42(8):1098-1108, 2008.

[3] M. Papageorgiou, C. Diakaki, V. Dinopoulou, A. Kotsialos, and Wang Y. Review of road traffic control strategies. Proc. of the IEEE, 91(12):2043-2067, 2003.

[4] M. Papageorgiou, H. Hadj-Salem, and J.-M. Blosseville. Alinea: A local feedback control law for on-ramp metering. Transportation Research Record, (1320):58-64, 1991.

[5] D. Pisarski and C. Canudas de Wit. Optimal balancing of road traffic density distributions for the cell transmission model. In Proc. of the 51th IEEE Conference on Decision and Control (CDC'12), page 6969 6974, Maui, Hawaii, USA, 2012.

[6] G. Como, E. Lovisari, and K. Savla. Throughput optimal distributed routing in dynamical flow networks. In Proc. of the 52st IEEE Conference on Decision and Control (CDC'13), Florence, Italy, 2013.

[7] E. Lovisari, C. Canudas de Wit, and A. Y. Kibangou. Data fusion algorithms for density reconstruction in transportation networks. In Proc. of the 54th IEEE Conf. Dec. Cont. (CDC'15), 2015.

[8] M. J. Lighthill and G. B. Whitham. On kinematic waves. ii. a theory of traffic flow on long crowded roads. Proc. of the Royal Society of London, Series A, Mathematical and Physical Sciences, 229(1178):317345, 1955.

[9] C. F. Daganzo. The cell transmission model, part II: network traffic. Transport. Res. B-Meth., 29B(2):79-93, 1995.

[10] M. Shahabi and A. Unnikrishnan. Robust hub network design problem. Transport. Res. E-Log., 70(0):356 - 373, 2014.

[11] A. Ehlert, M. G. H. Bell, and S. Grosso. The optimisation of traffic count locations in road networks. Transport. Res. B-Meth., 40(6):460 $-479,2006$.

[12] S.-R. Hu and H.-T. Liou. A generalized sensor location model for the estimation of network origindestination matrices. Transport. Res. C-Emer., 40(0):93 - 110, 2014.

[13] E. Lovisari, G. Como, and K. Savla. Stability of monotone dynamical flow networks. In Proc. of the 53st IEEE Conf. Dec. Cont. (CDC'14), pages 2384-2389, Dec 2014.

[14] C. Canudas de Wit, F. Morbidi, L. L. Ojeda, A. Y. Kibangou, and I. Bellicot. Grenoble traffic lab: An experimental platform for advanced traffic monitoring and forecasting. IEEE Control Syst. Mag., 2015.

[15] Centre national d'information routiére (CNIR). Website: http://www.bison-fute.gouv.fr/. 